\title{
A Novel Technique for Accuracy in Horizontal, Vertical, and Axial Bracket Placement in Orthodontic Bonding
}

\author{
Alka Banker ${ }^{1}$, Varshin Vala², Bhagyashree B Desai ${ }^{3}$
}

\begin{abstract}
Aim: The aim of the study was to develop a method which can help us achieve precise and accurate direct bonding (DB).

Background: Proper and accurate bracket position during orthodontic bonding is one of the main concerns for the clinician. Proper bonding reduces the effort to achieve good finishing by minimizing the wire bending. Unwanted tooth movements like unplanned tip, torque, and rotation are minimized, so the treatment time is shortened. Commonly used techniques for bonding brackets to teeth are DB and indirect bonding (IDB). Technique: A new technique involving the innovative use of tooth-shaped stickers was suggested to combine the advantages of both the methods. Conclusion: There was an accuracy in horizontal, vertical, and axial positioning of the brackets using this method.

Clinical significance: We found this as an effective method to achieve ideal bracket position by eliminating subjective bias and standardizing the bonding procedure, thus achieving better results in a shorter time by eliminating bonding errors.

Keywords: Accuracy, AutoCAD software, Direct bonding, Fixed orthodontic treatment, Orthodontic brackets, Precision, Tooth shape, Tooth stickers. The Journal of Contemporary Dental Practice (2021): 10.5005/jp-journals-10024-3190
\end{abstract}

\section{BACKGROUND}

Proper and accurate bracket position during orthodontic bonding is one of the main concerns for the clinicians. Proper bonding reduces the effort to achieve good finishing by minimizing the wire bending. Unwanted tooth movements like unplanned tip, torque, and rotation are minimized and so treatment time is shortened. Many studies have compared the two main techniques used for bonding, direct bonding (DB) and indirect bonding (IDB), and have found no significant difference between the two. ${ }^{1}$

DB is more commonly used as IDB has a few disadvantages, namely more laboratory time required, more steps involved so more chances of error, steep learning curve, difficulty in visualization of bracket placement, difficulty in removing excessive flash, and not easy to give proper pressure, so chances of voids and bond failure. However, DB is not without its own set of challenges and studies have found inaccuracies in bracket positions despite the operator's skill and also because of difficulty in accessing the posterior teeth. ${ }^{2}$

\section{TECHNIQUE}

A simple method was devised to achieve ideal bracket position with DB.

The bracket base was measured (Dentaurum equilibrium brackets), and cutouts were made of that size for each bracket. A basic stencil of tooth anatomy was used, and the cutouts for brackets were placed at a precise location using AutoCAD ${ }^{\circledR}$ software, according to the McLaughlin-Bennett-Trevisi (MBT) bracket placement chart. The size of the stencil was adjusted to get five tooth sizes, namely extralarge, large, medium, small, and extrasmall (Fig. 1).

This stencil was then scanned and printed on a plastic sticker sheet and laser cut.

Teeth were prepared for bonding, and after etching, the stickers were placed on the tooth such that the occlusal edges match (Fig. 2A). The sticker series which closely matched the tooth

\footnotetext{
*Laser Pro Spirit Machine
}

'Department of Orthodontia, Pulse Orthodontia, Ahmedabad, Gujarat,
India
${ }^{2}$ Unitedworld Institute of Design, Karnavati University, Gandhinagar,
Gujarat, India
${ }^{3}$ Department of Orthodontics and Dentofacial Orthopaedics, Faculty
of Dental Science, Dharmsinh Desai University, Nadiad, Gujarat, India

Corresponding Author: Alka Banker, Department of Orthodontia, Pulse Orthodontia, Ahmedabad, Gujarat, India, Phone: +91 9824002666, e-mail: bankeralka@yahoo.com

How to cite this article: Banker A, Vala V, Desai BB. A Novel Technique for Accuracy in Horizontal, Vertical, and Axial Bracket Placement in Orthodontic Bonding. J Contemp Dent Pract 2021;22(9):1076-1078.

Source of support: Nil

Conflict of interest: None

size was chosen. Now, primer was applied to the cutouts, and the brackets were bonded (Fig. 2B). Tooth stickers were then peeled off and any remaining part adhering to the brackets was removed using a scaler (Fig. 2C).

\section{Results}

When the bracket position was checked using a digital caliper, it was found that there was an accuracy in horizontal, vertical, and axial positioning of the brackets.

\section{Discussion}

To get the full advantage of the preadjusted appliance, it is necessary to have proper and accurate bonding of the brackets. Precise bonding may lead to a greater treatment efficiency and shorter treatment times. Both the commonly used methods of bonding, DB and IDB, have their own set of advantages and disadvantages. DB technique is more routinely used at present.

(O) The Author(s). 2021 Open Access This article is distributed under the terms of the Creative Commons Attribution 4.0 International License (https://creativecommons. org/licenses/by-nc/4.0/), which permits unrestricted use, distribution, and non-commercial reproduction in any medium, provided you give appropriate credit to the original author(s) and the source, provide a link to the Creative Commons license, and indicate if changes were made. The Creative Commons Public Domain Dedication waiver (http://creativecommons.org/publicdomain/zero/1.0/) applies to the data made available in this article, unless otherwise stated. 


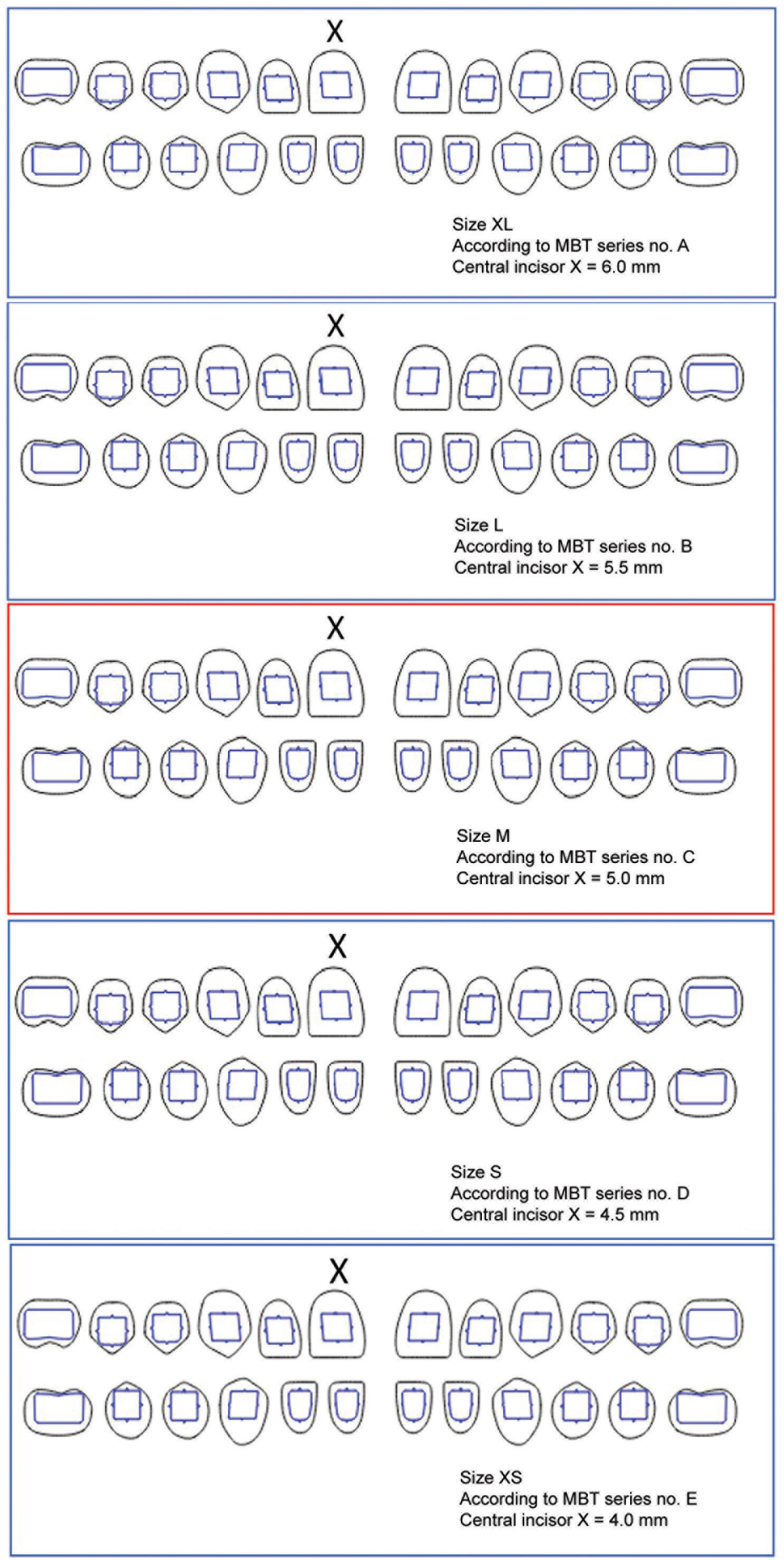

Fig. 1: AutoCAD drawing of tooth series in five sizes with bracket cutouts

Studies comparing DB with IDB concluded that DB had fewer bond failures, allowed easier flash removal, and the brackets were more closely fitted to the tooth surface with fewer voids. ${ }^{3}$ However, this technique depends on the operator's skill for precise bracket placement. Studies conducted to determine the accuracy of bracket placement with the direct bonded technique found a mean of $0.34 \mathrm{~mm}$ for the vertical discrepancies and a mean of $5.54^{\circ}$ for the angular discrepancies. ${ }^{2}$ In spite of using Boone's gauge, clinicians were prone to making mistakes during bracket placements. ${ }^{4}$ Though techniques involving the use of digital methods such as rapid prototyping and stereolithography are now available, they are not routinely accepted and are still in the future.

Thus, currently, there is no method that can give the advantage of a high degree of precision in bracket angulations and positions 

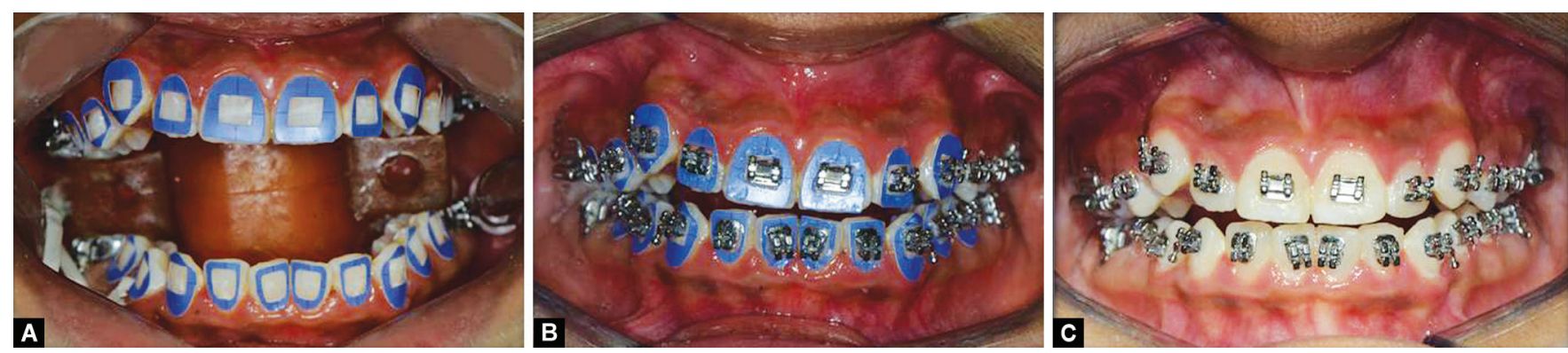

Figs 2A to C: (A) Stickers placed on tooth; (B) Bracket bonding in the cutouts; and (C) Stickers removed

along with ease of use, easy flash removal, and even pressure to achieve good contact between bracket base and tooth.

A simple method was devised in our clinic to overcome these problems. We found that this technique, involving the use of tooth stickers, solved many of the problems inherent in both DB and IBD techniques.

\section{ConcLusion}

Ideal bracket position can be achieved by eliminating subjective bias and standardizing the bonding procedure, using this efficient and effective technique. This method could be one more tool that can be added to a clinician's armamentarium for bonding procedures.

\section{Clinical Significance}

We found the following advantages in this technique:

- Precise placement of brackets, even to $0.25-\mathrm{mm}$ accuracy without the use of bracket positioning gauges or IDB trays.

- Easy visualization while placing the brackets.
- Easy to apply even pressure to avoid voids in bonding material which can lead to bond failures.

- Bonding can be done by auxiliary personnel as the bracket position is well defined.

- Easier to remove excessive adhesive flash.

- Deeper or flatter smile curve can be customized.

- Cost-effective with a minimal learning curve.

\section{References}

1. Aguirre MJ, King GJ, Waldron JM. Assessment of bracket placement and bond strength when comparing direct bonding to indirect bonding techniques. Am J Orthod 1982;82(4):269-276. DOI: 10.1016/0002-9416(82)90461-4.

2. Balut N, Klapper L, Sandrik J, et al. Variations in bracket placement in the preadjusted orthodontic appliance. Am J Orthod Dentofacial Orthop 1992;102(1):62-67. DOI: 10.1016/0889-5406(92)70015-3.

3. Zachrisson BU, Brobakken BO. Clinical comparison of direct versus indirect bonding with different bracket types and adhesives. Am J Orthod 1978;74(1):62-78. DOI: 10.1016/0002-9416(78)90046-5.

4. Mota Júnior SL, de Andrade Vitral J, Schmitberger CA, et al. Evaluation of the vertical accuracy of bracket placement with the Boone gauge. Am J Orthod Dentofacial Orthop 2015;148(5):821-826. DOI: 10.1016/ j.ajodo.2015.05.028. 